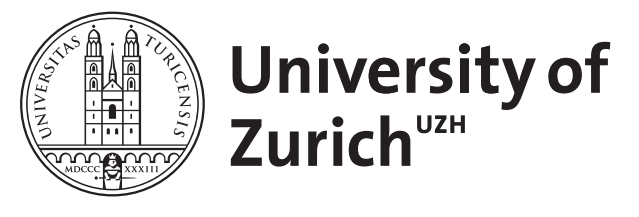

\title{
General Anesthesia and Fabry Disease
}

Krüger, Stefan ; Nowak, Albina ; Müller, Torben Christoph

\begin{abstract}
Fabry disease is an inherited X-linked disorder characterized by the absence (in men) or deficiency (in women) in -galactosidase A activity that causes a progressive accumulation of glycosphingolipids within lysosomes of cells of all the major organ systems. The subsequent organ damage that manifests in childhood and early adulthood presents a widely variable clinical picture of pain, hypertension, and cardiac, renal, nervous system, and lung dysfunction. We present 2 female patients with Fabry disease who required general anesthesia twice for gynecological and trauma surgery, respectively, and discuss their perioperative management based on new information in the medical literature.
\end{abstract}

DOI: https://doi.org/10.1213/XAA.0000000000000479

Posted at the Zurich Open Repository and Archive, University of Zurich ZORA URL: https://doi.org/10.5167/uzh-140901

Journal Article

Published Version

Originally published at:

Krüger, Stefan; Nowak, Albina; Müller, Torben Christoph (2017). General Anesthesia and Fabry Disease. A A Case Reports, 8(10):247-249.

DOI: https://doi.org/10.1213/XAA.0000000000000479 


\title{
General Anesthesia and Fabry Disease: A Case Report
}

\author{
Stefan Krüger,* Albina Nowak, MD,† and Torben Christoph Müller*
}

\begin{abstract}
Fabry disease is an inherited X-linked disorder characterized by the absence (in men) or deficiency (in women) in $\alpha$-galactosidase A activity that causes a progressive accumulation of glycosphingolipids within lysosomes of cells of all the major organ systems. The subsequent organ damage that manifests in childhood and early adulthood presents a widely variable clinical picture of pain, hypertension, and cardiac, renal, nervous system, and lung dysfunction. We present 2 female patients with Fabry disease who required general anesthesia twice for gynecological and trauma surgery, respectively, and discuss their perioperative management based on new information in the medical literature. (A\&A Case Reports. 2017;8:247-9.)
\end{abstract}

$\mathrm{F}$ abry disease, also known as Anderson-Fabry disease, is an inherited X-linked disorder characterized by the absence (in men) or deficiency (in women) in $\alpha$-galactosidase A activity that causes a progressive accumulation of glycosphingolipids within lysosomes of cells in all the major organ systems and progressive organ damage that first manifests in childhood or early adulthood. The widely variable clinical picture includes acroparesthesia, cornea verticillata, and abdominal pain in childhood; angiokeratoma, renal insufficiency, and electrocardiographic abnormalities in adolescence; and excessive daytime sleepiness, obstructive sleep apnea, hypertension, cardiomyopathy, renal failure, and stroke in adults. ${ }^{1-3}$ General anesthesia has been described in only 4 patients. ${ }^{4-6}$ We present 2 female patients with Fabry disease who required general anesthesia twice for gynecological and trauma surgery, respectively, and discuss their perioperative management based on the explosion of new information in the medical literature. ${ }^{1-3,7-10}$

\section{PATIENT 1}

A 37-year-old Caucasian woman, diagnosed with Fabry disease at age 24 during her workup for hypertension in 2003, presented for diagnostic laparoscopy and, subsequently, for laparoscopic resection of endometrial foci and the sigmoid colon with end-to-end anastomosis to treat her endometriosis. Her serum creatinine was $1.03 \mathrm{mg} / \mathrm{dL}$ (91 $\mu \mathrm{mol} / \mathrm{L})$ (elevated); her estimated glomerular filtration rate, $69 \mathrm{~mL} / \mathrm{min}$ (decreased). Because of the limited severity of her symptoms, she was not undergoing enzyme replacement therapy with $\alpha$-galactosidase A. ${ }^{1,10}$ Her hypertension was treated with metoprolol. Her history was negative for the 4 pain phenotypes of Fabry disease (pain attacks, pain crises, evoked pain/hyperalgesia, and chronic pain $)^{1,11}$ and obstructive sleep apnea. ${ }^{3}$

General anesthesia for both surgeries consisted of induction with fentanyl $3 \mu \mathrm{g} / \mathrm{kg} \mathrm{IV}$, propofol target-controlled infusion (TCI) $8 \mu \mathrm{g} / \mathrm{mL} \mathrm{IV}$, and rocuronium $0.6 \mathrm{mg} / \mathrm{kg} \mathrm{IV}$;

From the *Department of Anesthesia and Intensive Care, Kantonsspital Schaffhausen, Schaffhausen, Switzerland; and †Clinic of Medicine/ Endocrinology, UniversitätsSpital Zürich.

Accepted for publication November 15, 2016.

Funding: None.

The authors declare no conflicts of interest.

Address correspondence to Torben Christoph Müller, Kantonsspital Schaffhausen, Department of Anesthesia and Intensive Care, Geissbergstr. 81 8208 Schaffhausen, CHSwitzerland. Address e-mail to Torben.mueller@me.com.

Copyright (C) 2017 International Anesthesia Research Society

DOI: $10.1213 /$ XAA.0000000000000479 maintenance with propofol and remifentanil TCI; and additional analgesia with paracetamol, metamizole, and fentanyl IV. A lidocaine infusion was added to the maintenance regiment for the longer second surgery. ${ }^{7}$ Dexamethasone and ondansetron were administered to minimize postoperative nausea and vomiting. Neostigmine and glycopyrrolate were administered to reverse neuromuscular block in 1 case. Extubation was routine and uneventful, and there was no prolonged emergence or nothing unusual in her analgesic requirements.

\section{PATIENT 2}

A 67-year-old Caucasian woman, diagnosed at age 40 with Fabry disease during her workup for diffuse angiokeratomas, presented for osteosynthesis of her left ankle following a bimalleolar fracture and, subsequently, for removal of osteosynthetic hardware. She suffered from chronic neuropathic and myofascial pain, cardiomyopathy, hypertension, and renal insufficiency, but physical and echocardiographic signs of heart failure were absent. She was undergoing enzyme replacement therapy with agalsidase alfa $0.2 \mathrm{mg} / \mathrm{kg}$ every 2 weeks (Replagal; Shire Human Genetic Therapies, Inc). Her other chronic medications included aspirin, bisoprolol, candesartan, lercanidipine, metamizole, and pantoprazole.

For the first surgery, we induced anesthesia with fentanyl $3 \mu \mathrm{g} / \mathrm{kg} \mathrm{IV}$, propofol $150 \mathrm{mg} \mathrm{IV}$, and rocuronium $0.6 \mathrm{mg} / \mathrm{kg}$, and utilized desflurane and remifentanyl for maintenance. Paracetamol and metamizole were used for postoperative pain; dexamethasone and ondansetron to minimize postoperative nausea. Norepinephrine $2 \mu \mathrm{g} / \mathrm{min}$ and ephedrine were required to stabilize the blood pressure. For the second surgery, maintenance anesthesia was changed to propofol TCI for additional reduction of nausea because she experienced mild nausea after the first anesthetic. Continuous infusion of catecholamines was not required.

\section{DISCUSSION}

Fabry disease, also known as Fabry's disease, AndersonFabry disease, $\alpha$-galactosidase A deficiency, or angiokeratoma corporis diffusum, is an X-linked lysosomal storage disease first described simultaneously but separately in 1898 by dermatologists from Germany, Johannes Fabry, ${ }^{12}$ and the United Kingdom, William Anderson. The first sign described by Fabry and Anderson was the dermal manifestation called angiokeratoma, which can appear all over the body but most commonly manifests on the lower abdomen, 
buttock, and thighs. Now early-onset renal insufficiency is the most common early sign leading to the diagnosis.

The production of lysosomal enzyme $\alpha$-galactosidase A is reduced in Fabry disease because of mutation of the corresponding gene on the $\mathrm{X}$ chromosome. Accumulation of the glycosphingolipid globotriaosylceramide (Gb3) results and causes cellular dysfunction not only in several organ systems, mainly skin, kidney, heart, lung, and brain, but also in the gastrointestinal tract and cornea. The injured endothelial cells of small and large vessels and vascular smooth muscle cells cause dysfunction of the heart and brain leading to early onset of hypertension, concentric left ventricular hypertrophy without obstruction, and coronary heart disease, as well as vertebrobasilar artery signs and symptoms, excessive daytime sleepiness, and stroke. Small fiber neuropathy presenting as acroparesthesia of the hands and feet can occur as early as age $3 .^{1}$

The measurement of the $\alpha$-galactosidase A activity can be employed as a diagnostic tool in males. In females, the enzyme activity can be borderline or normal because of genotypic heterogeneity and random $X$ chromosome deactivation. Therefore, a genetic testing for pathologic mutations of the GLA gene is always required in females. The subsequent therapy involves the substitution of recombinant $\alpha$-galactosidase $\mathrm{A}$ as well as individual treatment of the symptoms, and it depends on the organs harmed.

The prevalence of Fabry disease is difficult to determine, especially in women. Switzerland, the country from which we are reporting, expects a prevalence of approximately 1:6000 to $1: 40,000$ (heterozygous women) and 1:40,000 to $1: 60,000$ (men). ${ }^{1}$ On the other hand, neonatal genetic screening leads to higher values. Italy reported that 30 per 100,000 babies carry the mutation, while Taiwan counted 80 per $100,000 .{ }^{13}$ Throughout all studies, men suffer more intense symptoms and faster progression of disease, as expected from the lack of a second, nonmutated $X$ chromosome. The wide variability of symptoms in women suggests a large number of them with $\alpha$-galactosidase A deficiency may go undiagnosed. In our cases, the symptoms leading to diagnosis were earlyonset arterial hypertension and renal insufficiency in the first patient and angiokeratoma in the second patient. Because of these relatively unspecific manifestations, the real prevalence of Fabry disease is expected to be higher in women than the prevalence that common knowledge indicates. ${ }^{13}$
In consideration of the various manifestations and differences in the involvement of specific organs, the preoperative assessment must identify the specific pattern of symptoms present in the individual patient (Figure). Especially, severe damage to heart, brain, lung, and kidney must be sought and either optimized preoperatively or controlled during anesthesia. ${ }^{6}$ This involves the adaption of the anesthesia to the surgery performed and to predictable problems like trauma (hyperalgesia), cardiac/pulmonary distress, and potentially prolonged elimination (renal insufficiency). The adoption of a perioperative pain treatment plan is certainly of high value for the patient. However, there is no specific protocol to follow as yet, and the individual conditions of both patient and surgery provide a challenge in defining a general approach. Some patients might undergo a nontraumatic surgery but suffer from chronic pain from their early childhood on. ${ }^{1}$ Others, as in our case 1 , have mild symptoms but the surgery is traumatic. Although there are very few data regarding Fabry disease and general anesthesia, as mentioned, there is even less concerning peri- and postoperative pain, perhaps indicating uneventful outcome and not much need for adoption of specific perioperative pain protocols. This thesis, however, certainly requires very careful consideration due to the lack of data, because we also adapted our treatment slightly by infusing lidocaine in 1 case.

In conclusion, we added 4 more anesthetics to the literature of patients with Fabry disease and brought the total to 8 . The general anesthetics in our patients were essentially uneventful because of preoperative identification of the medical issues experienced by patients with Fabry disease. Compared with equivalent patients without Fabry disease, we had to deal with early-onset arterial hypertension, hyperalgesia, cardiomyopathy, and renal dysfunction. No additional or extended monitoring was required in any of the cases, but that would have been different with more severe symptoms. Enzyme replacement therapy did not appear to interfere with any of the drugs we used. Therefore, agalsidase alfa therapy should be continued following regular prescription when undergoing general anesthesia. Fabry disease, depending on sex, age, and severity of the disease, shows a great variety of symptoms and signs that need to be looked for and assessed carefully before general anesthesia. However, patients with mild progression of the disease require only a few adjustments to keep them stable with a beneficial outcome.

\begin{tabular}{|c|c|}
\hline Organ involved & Symptom \\
\hline Skin & Hypohidrosis, Angiokeratoma \\
\hline Heart & $\begin{array}{l}\text { Hypertension, valvular dysfunction, arrhythmia, } \\
\text { conduction abnormalities, left ventricular } \\
\text { hypertrophy, heart failure }\end{array}$ \\
\hline Lung & Obstructive pulmonary disease \\
\hline Brain & Stroke, transient ischemic attack \\
\hline Peripheral nerves & $\begin{array}{l}\text { Acroparesthesia, hyperalgesia, tinnitus, heat } \\
\text { intolerance }\end{array}$ \\
\hline Kidney & Proteinuria, hyperfiltration, kidney failure \\
\hline Gastrointestinal tract & $\begin{array}{l}\text { Cramping, diarrhoea, bloating, nausea, } \\
\text { malabsorption }\end{array}$ \\
\hline Eye & Cornea verticillata \\
\hline
\end{tabular}

Figure. Main manifestations of Fabry disease (table). The tables shows the symptoms commonly diagnosed in patients suffering from Fabry Disease. Bold marks indicate anesthesiologic relevance of the symptom. 


\section{Written Consent}

We obtained the written consent of the patients permitting the publication of this case report based on their medical data. $\#$

\section{DISClOSURES}

Name: Stefan Krüger.

Contribution: This author helped complete this manuscript.

Name: Albina Nowak, MD.

Contribution: This author helped complete this manuscript.

Name: Torben Christoph Müller.

Contribution: This author helped complete this manuscript.

This manuscript was handled by: Raymond C. Roy, MD.

\section{REFERENCES}

1. Rodieux F, Pfister M, van den Anker J, et al. Unexplained peripheral neuropathic pain and/or stroke. Swiss Archives Neurol Psychiatry Psychotherapy. 2016;167:74-80.

2. Schiffmann R, Ries M. Fabry disease: a disorder of childhood onset. Pediatr Neurol. 2016;64:10-20.

3. Franzen D, Gerard N, Bratton DJ, et al. Prevalence and risk factors of sleep disordered breathing in Fabry disease. Medicine. 2015;94:52.
4. Watanabe H, Aoki T, Ono A. The anesthetic management of a patient with Fabry's disease. Masui. 1995;44:1258-1260.

5. Woolley J, Pichel AC. Peri-operative considerations for Anderson-Fabry disease. Anaesthesia. 2008;63:101-102.

6. Sorbello M, Veroux M, Cutuli M, et al. Anaesthesiologic protocol for kidney transplantation in two patients with Fabry disease: a case series. Cases J. 2008;1:321.

7. Politei JM, Bouhassira D, Germain DP, et al. Pain in Fabry disease: practical recommendations for diagnosis and treatment. CNS Neurosci Ther. 2016; 22:568-576.

8. Sené T, Lidove O, Sebbah J, et al. Cardiac device implantation in Fabry disease: A retrospective monocentric study. Medicine. 2016;95:e4996.

9. Seydelmann N, Liu D, Krämer J, et al. High-sensitivity troponin: a clinical blood biomarker for staging cardiomyopathy in Fabry disease. J Am Heart Assoc. 2016;5:e002839.

10. El Dib F, Gomaa H, Carvalho RP, et al. Enzyme replacement therapy for Anderson-Fabry disease. Cochrane Database Syst Rev. 2016;7:CD006663.

11. Uceyler N, Magg B, Thomas P, et al. A comprehensive Fabry-related pain questionnaire for adult patients. Pain. 2014;155:2301-2305.

12. Fabry J. Ein Beitrag zur Kenntnis der Purpura haemorrhagica nodularis (Purpura papulosa haemorrhagica Hebrae). Archiv Dermatol Syphilis. 1898;42:187-200.

13. Germain DP. Fabry disease. Orphanet J Rare Dis. 2010;5:30. 